MARK DARIUS JUSZCZAK

St. John's University

juszczam@stjohns.edu

\title{
WHEN DOES THE ANTHROCOSMIC MEDIA AGE BEGIN?
}

\begin{abstract}
The Google Lunar X Prize, which ends on December 31, 2017, has the potential to generate the first true instance of anthrocosmic media - media intended for consumption in outer space, which serves as a form of capital for an emergent autonomous field in the same. Winning the bonus segment of the X Prize consists of creating multimedia (photography or video) of one of the Apollo Landing Sites on the lunar surface. Both the terms of the creation of that media, and the media itself, are the first instances of agents acting to establish autonomy on the lunar surface - controlling how artifacts are accessed, used and represented. This paper is an exploration of the conditions required for the emergence of an anthrocosmic media age - an age where agents use media as a form of power in outer space to establish autonomy in fields that are also in outer space.
\end{abstract}

Keywords: anthrocosmic media; space; lunar Surface

\section{Introduction}

Anthrocosmic Media: Media intended for consumption in outer space. Media created in order to influence or alter the social capital of agents situated in an autonomous field in outer space.

Ever since the Soviet Union launched the Sputnik satellite on October 4, 1957, human civilization has been a creator of media in space for consumption on Earth. Sputnik I did not merely circle the Earth. A few minutes after entering orbit, Sputnik began transmitting at 20.005 and $40.002 \mathrm{MHz}$. Its signal was monitored and picked up by not only the Soviet Union and the United States, but by amateur radio operators all over the world (Didlake 2007).

Although Sputnik I's orbit decayed and Sputnik burned up in the atmosphere three months later on January 4, 1958, it ushered in the age of outer space media: media created in space for consumption on Earth; space theater, in effect. Space media has, since then, proliferated to an extent perhaps almost unimaginable in 1957. We take for granted that we can download on our cellphones images from the surface of Mars, or that we can watch the Apollo 11 Moon landing on Youtube, or that we can examine images of galaxies far, far away.

Outer space media is, however, a content based category. It is defined and limited by the content of the subject. To state the obvious, it does not reflect the status or interests of agents, as a social class, who are themselves autonomous and 
non-terrestrial humans. Astronauts are explorers, not citizens of space. Astronauts do not speak of space as 'home', nor do they seek to exercise sovereignty in any way in outer space. Quite the contrary, the presence of humans in outer space has almost exclusively been as 'theater' for humans on Earth and as a cultural product meant to reinforce the existing positions of Earth bound agents.

Even the personnel aboard the ISS (International Space Station) are transient guests of a series of inter-locking modules floating above the Earth. They do not seek to rebel against their masters on the ground. There is no struggle for autonomy or power between Earth and Orbit or among the personnel of various countries present on the ISS. Instead, there is, by design, an existence of transient, technical consensus and cooperation. Although these personnel may create media on board the ISS for consumption by other ISS personnel, we would not call that media a cultural product of outer space. It does not represent a unique social class in space exercising an independent form of power, nor a unique cultural or social form of capital. It is a practical necessity for survival in an environment that is very explicitly transient.

In other words, existing media about space is not intended for consumption in space, precisely because it does not reference or alter an existing state of autonomous cultural production in space or a balance of cultural or social capital. And yet, if we look at a number of evolving technologies (namely those focused on lowering the cost of access to Earth orbit and those focused on increasing the velocity of spacecraft so as to enable both a near-interplanetary human civilization and a capacity to visit other star systems) it appears that a permanent autonomous human presence beyond Earth is inevitable in this century.

Given that permanent human presence beyond Earth, we can imagine, in some not-too-distant future, on the first permanent human colony in outer space (either in orbit, on the Moon or on Mars), a desire among colonists for self-determination and the creation of media and culture that will seek to define a clearly independent and new type of power as well as a new type of human social structure. Will it be the Lunar Revolution of 2076? Will it be the Martian Colonists Revolution of 2091 ? We can imagine it because we have seen it before. The Declaration of Independence in 1776 is one of the more prominent examples of this expressed desire for selfdetermination and autonomy. And it is but one of many examples throughout history of the expressed desire for self-determination, and the struggle (both through violence and through the production of unique culture and media) to acquire and persist in that self-determination.

The question I would pose, in the context of the Declaration of Independence, signed on July 4, 1776, is: when did the age of American media begin? Did it begin with that document? Did it began when American colonists won the war for Independence and signed the Treaty of Paris in 1783? Did it began when, prior to 1776, a first colonist wrote in a colonial newspaper of him or herself as an 'American' and not as a British subject of the King's colony? Or is the boundary fuzzy? And, is it only in hindsight that we can look back and say that a distinct period of autonomous cultural production began at a certain time? Does the present blind us and bind us?

Durkheim said, "In each one of us, in differing degrees, is contained the person we were yesterday, and indeed, in the nature of things it is even true that our past personae predominate in us, since the present is necessarily insignificant when 
compared with the long period of the past because of which we have emerged in the form we have today" (Durkheim 1977: 11). Durkheim identifies, indirectly, an interesting problem in classification: that of defining boundaries of cultural production. According to Durkheim (and Bourdieu), the present cannot be separated from the past. But this presents a problem in the research of media as cultural production. Because at some point, for the purpose of inquiry, a boundary must be identified.

This is most certainly true if we accept Bourdieu's premise that media, as cultural production, must be analyzed within a certain field and that field must have a certain evident degree of autonomy. That autonomy need not be total (such as that bestowed upon a sovereign nation). But it must be evident. And, if there is a field, then there must also be a non-field or a way of defining the boundary between fields, or the point at which that locally defined power seizes to function.

The fact that outer space media is not explicitly, in research and literature, constrained by the place of its origin, is informative. Whether a given type of media about space is produced on Earth (via telescopes or other types of observational instruments) or produced in outer space, it does not particularly matter from the perspective of field, as defined by Bourdieu. According to Pierre Bourdieu's theory of cultural production (Bourdieu 1993) all technologies and modalities of culture (inclusive of the arts, literature, media, monuments, etc.) can only be effectively analyzed through the model of a field: defined as the setting in which agents and their social positions are located (Bourdieu 1993). This setting, in addition, has certain properties that are critical in this context: it must display some measure of autonomy and power must be locally defined with respect to the agents within that field. Both of these elements of field will be discussed extensively below.

The phrase "are located" is significant for two reasons. First of all, it implies, but does not state, what is obvious on Earth: a permanence or semi-permanence of geographic or cultural power imbued with a local history. A journalist for the New York Times who has traveled to attend a summit in Belgium does not suddenly become Belgian. Neither does a Russian journalist who travels to the United States to conduct a field study on American fashion preferences become an American. Although they are "located" in a given geographic space that is different than their own, they do not become "of that space". We do not use words like transient or temporary to signify their social position as agents.

Second of all, it implies the ability to delineate location: to say that this field exists here but not there. And that notions of here and there are not explicitly categories of geography. They are, rather, categories of autonomy, true to the idea of the field itself. They are also categories of classification. Although Bourdieu may not have intended it to be the case, the concept of field must also include in it a way to classify where one field ends and another begins.

The cultural history of humans on Earth is one of overlapping boundaries and histories. Field boundaries are fuzzy because every revolution, every significant change of power and status is burdened with the prior history and context of its agents. To put this in the context of our prior example: the signing of the Declaration of Independence on July 4, 1776, may have created among the colonists the feeling that "we are now Americans" and the products of our culture are "American". But it does not mean that they were no longer British. Quite the contrary, the society and unique culture of what would become the United States 
was only possible because those first citizens of the United States were born and raised as British subjects. What happens, however, when humans enter a space that is almost entirely without history and without context?

The Apollo 11 lunar landing, for example, and landings by subsequent Apollo missions, brought humans to the Moon, but did not alter their social position as agents of cultural production. Neil Armstrong stepped onto the lunar surface as an American. And he and Buzz Aldrin departed quickly - as Americans. This is obvious, but its counter-point needs to be stated because of its broader significance: although humans have been on the Moon and have lived in space for periods of time on the International Space Station, they have not shed their social position as agents of cultural production bound to specific cultural and geographic identities on Earth. They did not acquire autonomy on the Moon as citizens of a lunar government. Instead, what they did was almost entirely space theater - a cultural production meant to reinforce the power of respective agents on Earth.

Even on the International Space Station, the production of material and media culture on the part of astronauts is largely bound to the respective cultures of their home countries. "ISS missions are fully international. Each crew contains at least one astronaut and one cosmonaut, and the station itself contains both Russian and American segments. Russian and American mission control centers jointly direct the operations, and Russian and American science experiments are given equal priority" (Ritsher 2005). Although there is extensive cooperation and collaboration, the existing structure of the work and the transience of ISS personnel suggests that, up to the present moment, the production of media aboard the ISS is principally a function of scientific and technical projects. This does not mean that cultural differences do not exist, or that they do not affect the work of personnel aboard the ISS. "Several surveys have highlighted the wide range of cultural issues that are likely to affect international space crews regardless of the particular nationalities involved. Some researchers posit that cultural contrasts can become magnified during missions" (Ritsher 2005). Rather it means that those differences are not directed to meaningfully affect either a desire for or attempt at autonomy, with power locally defined on the ISS itself. At the moment that space stations develop a unique form of currency and use it to conduct business with other space stations, it is certain that an autonomous field will occur with its own local definition of power.

Whatever the differences are, however, they are not differences in field, as defined by Bourdieu. And that is the point. That up to the present moment in time, there has not yet been a field that is uniquely and permanently in outer space; outer space as a setting in which agents and their social positions are located and, by consequence, a place where agents desire to be autonomous creators and consumers of media and culture for the purpose of altering and maintaining a certain position of power - in outer space.

It is my thesis in this paper that that is about to change. That we are about to enter an age of anthrocosmic media - where autonomous fields are created by agents situated in outer space precisely because they have cultural and social positions in outer space that must be protected or defined; precisely because they are about to be encountered.

The Google Lunar X Prize, specifically, has, inadvertently, created the first meaningful moment where agents, as both creators and consumers, are situated in 
outer space - and where the opportunity to exercise autonomy exists. This paper is an in-depth examination of the Google Lunar X Prize, from the perspective of Bourdieu's field theory, and the implications for the prize on the emergence of a distinct field, that of anthrocosmic media - media meant for consumption by agents in outer space, because it affords them the opportunity to acquire autonomy. We are about to enter the Anthrocosmic Age - where we, as humans, are not only technologists visiting space, but creators and consumers of media and culture in space for others in space.

In this paper I will first review Bourdieu's theory, with a specific focus on applications of field theory in situations where the settings of agents and their social positions are emergent (absent of significant historical contexts to formulate rules and norms of cultural production). I will then look, in detail, at the Google Lunar X Prize, from the perspective of the implications of its success on the first instance of a struggle between agents with respective vested social and cultural interests on the lunar surface. It is my hope to demonstrate that, given the tangled history of cultural and heritage status of the Apollo Landing Site, the successful completion of the Google Lunar X Prize will be the first true instance of anthrocosmic media - of agents with a situated and permanent interest in outer space producing culture and media to alter the balance of power and capital with respect to those interests.

\section{Part 1: Bourdieu's Field Theory}

Bourdieu's field theory presupposes that, for a specific agent and product of culture, there is a historical context to position and capital. "The position of each particular agent in the field is a result of interaction between the specific rules of the field, agent's habitus and agent's capital (social, economic and cultural). Fields interact with each other, and are hierarchical: most are subordinate to the larger field of power and class relations" (Bourdieu 1993). In addition, fields display certain characteristics: they are autonomous and they retain a locally defined form of power. It may help to think of the concept of a field as a "stage": actors and actresses are present. Within the logic of the play a distinct form of power emerges. That power can be economic, social, familial, religious, artistic, athletic, etc. And that form of power is, to some measure, autonomous.

Although Bourdieu frames his theory of fields largely in the context of art and literature, it is equally valid in the context of media. Bourdieu's model of the analysis of media is significant because it situates that analysis in the agents that are its producers and consumers. Without it, we are left with a comparative analysis alone or a textual analysis alone. Situating media and other modalities of cultural production within a particular position in a broader field allows for a more comprehensive type of analysis; an analysis that is centered on media (and, more explicitly, the meaning of media) as the product of friction and conflict. In other words, media acts as a form of capital; as a way to express, retain or acquire power. Bourdieu explains, "Every position, even the dominant one, depends for its very existence, and for the determination it imposes on its occupants, on the other positions constituting the field; and that the structure of the field, i.e. of the space of positions, is nothing other than the structure of the distribution of the capital of specific properties which governs success in the field and the winning of the external or specific profits which are at stake in the field" (Bourdieu 1993). And, if 
there are successes measured by the acquisition or persistence of modes of capital, then there are also losers. "The literary or artistic field is a field of forces, but it is also a field of struggles tending to transform or conserve this field of forces" (Bourdieu 1993).

Fundamentally, this is a Darwinian analysis of the production of culture, inclusive of media. In other words, according to Bourdieu, any type of cultural production (art, literature, media) has, as its ultimate purpose, the acquisition or persistence of power of that producer of culture. Media is neither made nor consumed without the intention of acquiring capital, increasing power or maintaining a dominant position in a respective field.

What this means practically is that the consumption of media can only be analyzed if several criteria are met: that the field is at least somewhat autonomous; that there exist at least two agents within a field; that the two agents represent varying degrees of dominance within a field that is common to both of them; and, that the agents agree on the form of power that is used within the field to define dominance or subordination.

According to Bourdieu, "The meaning of a work changes automatically with each change in the field within which it is situated for the spectator or reader" (Bourdieu 1993). By extension, a work only acquires meaning as media (as opposed to technology or tool) when a change in the field within which it is situated occurs - and that change has repercussions for the social capital of the respective parties in that field. The existence of a conflict that changes the social capital of the respective agents within a field is, therefore, a precondition for the existence of media - as cultural capital - in that field. Otherwise, the media may exist in a given physical space but may not belong to that space.

Let us take an extreme hypothetical example to understand the significance of this conclusion. Imagine that a photograph, as evidence, exists of a member of the Parliament in Norway committing a theft. There is only one photograph. And, for some reason, that photograph is lost. It is discovered a few days later in the suitcase of a school teacher in Nebraska. Although that media is in a different physical space (Nebraska), its meaning and power is only relevant in the place of its origin (Norway). And, as such, it is not considered media in Nebraska. It is, however, media in Norway. That is where the field of its agents are situated.

The same is true of outer space media, as opposed to anthrocosmic media. If we extend the same hypothetical example to this variance, it will be immediately clear. The example is the first footprint of Neil Armstrong as he stepped on the Moon. Let us assume for the moment that that first footprint was neither erased by the other footprints Neil Armstrong and Buzz Aldrin took as they walked around the Apollo 11 Landing Site nor by the blow back of the blast as Eagle, the Lunar Ascent Module, departed from the surface of the Moon (Spennemann 2007). As a result, that first footprint is presumed to remain intact. The surface of the Moon is seismically dead and, with the possible exception of a meteor impact, that footprint is, for all practical purposes, preserved eternally (Colwell et al. 2007).

The footprint, however, has a "symbolic significance". That significance is relevant to agents on Earth, most notably, those in the United States that have sought to either preserve or lay claim to it as a monument of American (and human) achievement. According to Bourdieu, this is because that footprint represents "A present past that tends to perpetuate itself into the future by reactivation in 
similarly structured practices... This embedded history, internalized as second nature and so forgotten as history is the active presence of the whole past of which it is the product" (Bourdieu 2001). While agents on the lunar surface may exert some autonomy in their actions, with respect to those agents who first left those footprints, that partial autonomy may be significant enough to pass the "test" of being a field - and of demonstrating the first instance of anthrocosmic media.

A critical factor in that "test" is the potential for conflict. At the moment when that footprint becomes accessible, and where a certain agent has the power to alter, control, manipulate or represent that footprint in order to acquire a form of social or cultural capital, that footprint becomes media. To quote Bourdieu, "The state of the power relations in this struggle depends on the overall degree of autonomy possessed by the field, that is, the extent to which it manages to impose its own norms and sanctions on the whole set of producers, including those who are closest to the dominant pole of the field of power" (Bourdieu 1993). In practical terms, the moment that someone can change that footprint on the Moon, is the moment that someone can impose norms. Although there have been numerous discussions about the status of the Apollo Landing Sites from a heritage and cultural preservation perspective, those discussions are meaningless. They can neither impose norms on the site itself, nor can they affect that footprint as media. That can only happen when a particular agent has the power to cross the boundary of that site. The boundary of the field (in its most literal and figurative sense here), as Bourdieu reminds us, is a "stake of the struggle" (Bourdieu 1993). The first and most significant example of the emergence of anthrocosmic media, the first true stake of the struggle, is, perhaps, about to occur with the launches of the finalist teams of the Google Lunar X Prize.

\section{Part 2: A Field Approach to the Google Lunar X Prize - Bonus Prize}

Google's Lunar X Prize, which offers up to $\$ 30$ million in prize money for a successful private launch to the Moon by the end of 2017, contains one specific bonus prize component that is of significance to field theory. Although the successful outcome of the bonus prize component is a media broadcast from the lunar surface, it is, at the same time, by accident of its design, one of the most likely forerunners for the emergence of a truly anthrocosmic media age. This is because the conditions of the bonus prize component create the potential for a conflict that is rooted in cultural and media artifacts that are both on the Moon and only intended for consumption on the Moon.

The general mission requirements for claiming the Lunar X prize are: landing (a team must land its craft on the surface of the Moon after providing advance notice of its launch and intended landing site), mobility (after landing, a team must move its craft a distance of at least five hundred meters below, on, or above the lunar surface along an interesting path in a deliberate manner) and mooncast (a team must transmit two "mooncasts" from the surface of the Moon) (X Prize 2017). The bonus prize component that is of significance to the field of media sociology is the Apollo Heritage Bonus Prize. This is a $\$ 4$ million prize that will be awarded to a team that can produce an Apollo Heritage mooncast from the site of Apollo 11, 12, 14, 15, 16, or 17 (X Prize 2017).

This $\$ 4$ million bonus prize is the most significant component of the Google Lunar X Prize from the perspective of the emergence of fields in Outer Space. To 
understand why, we have to first look at the general context of the Apollo 11, 12, 14, 15, 16 and 17 Landing Sites from a heritage and preservation perspective.

\section{Apollo Landing Sites as the First Heritage Sites Outside of Earth}

Ever since the late 1980's, the question of the future of the Apollo Landing Sites has been investigated by a number of scholars and agencies. At the heart of this question is the issue of status: what are the Apollo Landing Sites and who has jurisdiction over them? Are they World Heritage Sites? Are they derelict property of the U.S. Government? Are they metal bits and space trash intended for a future salvage yard on the Moon? Different groups, over the past several decades, have proposed different models for jurisdiction and management. Both because of proximity (to Earth) and because of the large number of small artifacts that could relatively easily be transported back to Earth. "The largely metal components of the Eagle's descent stage and the various scientific instruments left behind by the astronauts are reasonably robust but could be targeted by future souvenir hunters or salvage operators" (Fewer 2007).

One of the first proposal was a designation of "UNESCO's World Heritage status [that] could be applied to all spacecraft landing and crash sites on the Moon and Mars" (Fewer 2007). The problem with Earth construct designations, however, as Spennemann and other scholars have pointed out, is that of "enforcing protection" (Fewer 2007). The second problem is that we do not know what artifacts and media are explicitly present. Technically, even the waste generated and discarded by the Apollo landing teams is a form of cultural product. In December 1999, "New Mexico State University's Lunar Legacy Project - comprising an interdisciplinary team led by Beth O'Leary - received a grant... to compile an inventory of 106 items associated with Apollo 11 that were left behind at Tranquility Base" (Fewer 2007). This initial inventory is a start. Being able to exercise power over a space invariably also means knowing and being able to catalogue what it contains. The intense push, for example, of the French monarchy to create an accurate map of France is an excellent example of this in practice. To know what a thing, or set of things is, in detail, is also, in part, to exercise power over those things. However, an inventory is not enough. There must also be a capacity to regulate and use that inventory. Which brings us to an interesting point, in the context of Bourdieu: that any designation may be meaningless unless there is both a capacity to exercise power in the name of that designation and, also, a capacity to restrain certain types of actions. While this is relatively easy on Earth, it is almost impossible in outer space at this moment.

Thus, the current status of the remnants of the Apollo program on the Moon, however well-defined on Earth, does not constitute a field in space. There is no enforcement. There is no autonomy that is space-based. And there are no agents in space. "While a future intra-solar state system is difficult to imagine, international law is extended into space via the Outer Space Treaty and its sister treaties and agreements" (Hearsey 2009). The OST (Outer Space Treaty) "prevents any nation from claiming land on the Moon. Because of preservation methods on Earth, the inability to own land makes preservation of the Apollo Landing Sites extremely complicated. The Apollo Sites present a difficult legal situation because they are on land that has been internationally agreed upon not to be controlled by any state, 
and the preservation and management of the site would prove to be extremely difficult" (Reynolds 2012).

One of the limits to the current model is that the OST is an agreement between nations. One of the key gaps in current treaties about the usage of outer space is that they have been written up and agreed to by nation-states. This is logical, in the context of the available technology at the time. It appeared that the only agents in Outer Space would be agents designated by and representing states. Nowhere is this more evident than on the Moon. "The majority of artifacts resting on the lunar surface by the Apollo Astronauts all have one thing in common. They were brought to the Moon by Americans, and are still the U.S. Government property" (Reynolds 2012). One could further argue that the Moon landing was a performance of power by the government. It was, as well, "a performance of national identity. The lunar landings simultaneously created, enacted, and naturalized U.S. nationalism" (Fischhoff 2008).

The OST does not specify what the limits and rights of individuals and corporations are, however. And, as such, there is a non-state actor loophole in the current way in which humans regulate and attempt to govern outer space. In essence, the only way to know what the real exercise of power in outer space looks like, is to attempt to exercise as a non-state actor. Which is exactly what the Lunar $\mathrm{X}$ Prize is attempting to do by December 31, 2017.

\section{The Autonomy Threshold and the Lunar X Prize}

At its face value, the bonus component of the Lunar X Prize appears to simply be a technological hurdle: a private company has to land a robotic rover on the surface of the Moon in such a way that the rover can operate, can access one of the Apollo Landing Sites and can transmit media proof, in the form of photographs and video, that it has been at the site.

However, the robotic lander is operated by a private company, not a government. It is not subject to the OST or to other treaties. The lander is remotely controlled, but, because of the lag time between Earth and the Moon, that control is not total and cannot guarantee complete performance as dictated by the robot owners. That means that the robot lander has the power, whether intentional or not, to alter that Landing Site. It can accidentally knock over a flag. It can roll over a footprint. It can destroy debris that may be of significant historical or scientific value. Its signal, in theory, can also be intercepted by a rogue force that can hold the Landing Site hostage. How much would the U.S. Government be willing to pay to prevent the rover from destroying the Apollo 11 "first footprints"? How much would the U.S. Government be willing to pay to prevent the rover from destroying sensitive equipment or taking pictures that the government may not want publicized?

The real question is this: does the power to alter a cultural site and to control the media that comes from that site constitute a measure of autonomy significant enough to assert that there is, in fact, a new field of power? This is the central question of the paper. This is not a question of a rover landing on the Moon. Rovers have landed on Mars, and those rovers have not created a field of power in so doing. This is explicitly about two things: the existence on the Moon of artifacts that have a significant cultural and symbolic value to humans and the power to both represent and alter those artifacts and, in so doing, to create a new type of media: 
an "anthrocosmic media" that has, at its core, an emergent autonomy that is not of the Earth. Archaeologist Alice Gorman, "Identifies the fact that, although not explicitly autonomous (with respect to their Earth-based monitors) both NASA and the Astronauts of the Apollo Program created a distinct cultural history of space exploration as a decidedly American act" (Fewer 2007).

That act is about to be subject to the expressed desire of potentially nonAmerican non-state actors. Let us consider this in the most benign and economic form, to understand exactly why this specific instance may serve as the emergence of a field in outer space - a distinctly anthrocosmic media. The power of the rover, and its operators, to photograph is also the power to choose what to photograph and how to photograph. As we know from the Apollo 8 mission, and that famous photograph of Earth from the Moon, a single picture can influence millions of people and can have outsized consequences. The right photograph at the right place on the lunar surface can spurn a massive push for lunar tourism - or destroy it in its infant tracks.

I would argue that the anthrocosmic media age begins at the moment that a rover on the Moon creates media about the Apollo Landing Sites. This is because the creation of media is also about exclusion. What is documented and what is hidden. And that is an expressed action of an autonomy. No one, but the operator of that rover, can control what the rover does. What it documents and how it documents it. And, at that moment, a nascent, but new, field is created. Because there are agents creating media in space for consumption in space; for control over new forms of heritage, new forms of tourism, new forms of economy and new forms of culture. It is a performance and its meaning: "Whether it is a concert dance or a step on the moon's surface, is not created exclusively in the time and space of the performance. The symbolic capacity of any embodied performance depends upon the practices of embodied meaning making which precede and follow it. Performance can be understood as rising out of a vast field of materialized meanings and embodied powers" (Fischhoff 2008).

Where there is selection in performance, there is autonomy. And where there is autonomy there is power. Bourdieu's field theory is rooted in a model of agents that act within spaces that are rich with history and context. Although he may not have anticipated the usage of his theory to predict the emergence of a new class of media, namely anthrocosmic media, it is uniquely fitted to the task. That is because the first likely instance of its production can only occur in the singular accessible place in outer space that is already rich with historical and cultural meaning: the Apollo Landing Sites.

\section{Conclusion}

The United States Congress just passed, in July 2017, H.R. 2810 (Fiscal Year 2018), the National Defense Authorization Bill. Section 16XX of the bill mandates the "Establishment of Space Corps in the Department of the Air Force. This section would authorize the creation of a Space Corps within the Department of the Air Force and require the Secretary of the Air Force to certify its establishment by January 1, 2019" (H.R. 2810 2017).

If there is any question related to the formation of fields of power in space, the decision, by the United States Government to create a dedicated Space Corps within the Defense Department should put that question to rest. The anthrocosmic 
age of media is about to begin. Humans are about to become creators of media in space, for consumption in space, with the intent of exercising power in space. Media is going to be but one of the tools of that new autonomy. But it will be one of the most powerful ones. It is my contention that the Anthrocosmic Age (the Age of Humans in space as creators of culture and media) will most likely begin when the Apollo Landing Site bonus component of the Google Lunar X Prize is won. I believe that we are on the verge of a new field of media studies: not media from outer space, but media for outer space.

\section{REFERENCES}

Bourdieu, Pierre. 1993. The Field of Cultural Production. Cambridge, UK: Polity Press.

Bourdieu, Pierre. 2001. Structure. Habitus. Practices. Modern social theory. St. Petersburg: Aletheia.

Colwell, J. E., Batiste, S., Horányi, M., Robertson, S., and Sture, S. 2007. Lunar surface: Dust dynamics and regolith mechanics. Reviews of Geophysics 45(2): 126.

Durkheim, Emil. 1977. The Evolution of Educational Thought. London: Routledge \& Kegan Paul.

Fewer, G. (2007). Conserving Space Heritage: The Case of Tranquility Base. Journal of the British Interplanetary Society 60(1): 3-8.

Fischhoff, Rachel Q. 2008. Locating the Astronaut Body in Space. Honors Theses 128, http://wesscholar.wesleyan.edu/etd_hon_theses/128

Hearsey, Christopher M. 2009. Universal conservationism: a proposal to establish world heritage sites beyond Earth. A/AA Space 2009 Conference Proceeding, https://papers.ssrn.com/sol3/papers.cfm?abstract_id=1507252

H.R. 2810 (2017). 115th Congress, Retrieved from: https://www.congress.gov/bill/115th-congress/house-bill/2810/actions

Ralph H. Didlake, KK5PM; Oleg P. Odinets, RA3DNC. 2007. „Sputnik and Amateur Radio". American Radio Relay League. Archived from the original on 11 October 2007. Retrieved 26 March 2008.

Reynolds, Joseph P. 2012. One Small Step: An Analysis of International Space Law and How it Effects Historic Preservation. All Theses 1346, http://tigerprints.clemson.edu/all_theses/1346/

Ritsher, J. B. 2005. Cultural factors and the international space station. Aviation, Space, and Environmental Medicine 76(6): B135-B144.

Spennemann, Dirk H. R. 2007. Extreme cultural tourism from Antarctica to the Moon. Annals of Tourism Research 34(4): 898-918.

X Prize. 2017. Google. Retrieved from: http://lunar.xprize.org/about/guidelines 